\title{
Identification of etiologic agents of recurrent vulvovaginal candidiasis in patients from Isfahan, Iran
}

\author{
Kiana Shirani ${ }^{\oplus}{ }^{\oplus}$, Zahra Allameh ${ }^{2}$, Azadeh Moshtzan ${ }^{3 *}$ \\ ${ }^{1}$ Acquired Immunodeficiency Research Center, Isfahan University of Medical Sciences, Isfahan, Iran \\ ${ }^{2}$ Department of Obstetrics and Gynecology, School of Medicine, Isfahan University of Medical Sciences, Isfahan, Iran \\ ${ }^{3}$ Department of Infectious Disease, School of Medicine, Isfahan University of Medical Sciences, Isfahan, Iran
}

\section{*Correspondence to \\ Azadeh Moshtzan, Email: \\ drmoshtzan@gmail.com}

Received 7 June 2020

Accepted 20 Aug. 2020

Published online 10 Sep. 2020

Keywords: Recurrent Vulvovaginal candidiasis Candida albicans, Candida non-albicans

\section{Abstract}

Introduction: Occurrence of vulvovaginal candidiasis (VVC), as a common condition in women of childbearing age, is increasing all over the world as a result of extensive use of antibiotics and antifungal drugs.

Objectives: In the present study to gain the up-to-date information on involved species and the prevalence of the recurrent vulvovaginal candidiasis (RVVC) in Isfahan, Iran, we assessed the etiologic agents of aforementioned disease in women referred to the Al-Zahra hospital (Isfahan, Iran). Furthermore, we surveyed the possible relationship between age, education and marital status with prevalence of albicans and non-albicans candidiasis. Patients and Methods: Our study was conducted on subjects who were admitted to the gynecology and midwifery clinic between September 2017 and August 2018 and had clinical presentations of vulvovaginitis. Sampling of 100 women was done using sterile swab. Samples were transferred to the hospital laboratory for cellular and molecular investigations.

Results: Candida albicans was the main pathogen involved in the pathogenesis of RVVC and Candida glabrata is the second most common pathogen. Moreover, none of the 100 cases that we tested were infected with Candida krusei. We found a meaningful relationship between age and RVVC triggered by Candida albicans $(P<0.05)$ but there was no significant relationship between age and RVVC that triggered by non-albicans fungi. There was no meaningful correlation between levels of education, number of children and types of delivery with RVVC that infected by various albicans and non-albicans candidiasis $(P>0.05)$.

Conclusion: Candida albicans was the main pathogen involved in the pathogenesis of RVVC in Isfahan while Candida glabrata is the second most common pathogen. Despite their high cost, molecular methods have high value in accurate diagnosis of the RVVC.

\section{Introduction}

Occurrence of vulvovaginal candidiasis (VVC), as a common condition in women of childbearing age, is increasing all over the world as a result of extensive use of antibiotics and antifungal drugs $(1,2)$. It has been reported that $70 \%$ women experience aforementioned condition during their lifetime (3). The other epidemiological studies have reported that between $6 \%$ and $8 \%$ of women will develop recurrent vulvovaginal candidiasis (RVVC) that diagnosed by the occurrence of at least four episodes of VVC in previous 12 months $(4,5)$. Occurrence of RVVC is associated with a meaningful morbidity in females in all societies and its management is a puzzling issue for infectious disease specialties. In the past years, occurrence of RVVC was related to menopause and associated hormonal alterations, but today, the use of hormones replacement has increased its prevalence, however the issue is open to discussion $(2,6)$. Aforementioned

\begin{abstract}
Key point
Occurrence of vulvovaginal candidiasis (VVC), as a common condition in women of childbearing age, is increasing all over the world as a result of extensive use of antibiotics and antifungal drugs. In the present study to gain the up-to-date information on involved species and the prevalence of the recurrent vulvovaginal candidiasis (RVVC) in Isfahan, Iran, we assessed the etiologic agents of aforementioned disease in women referred to the Al-Zahra hospital (Isfahan, Iran). Based on our results Candida albicans was the main pathogen involved in the pathogenesis of RVVC in Isfahan and Candida glabrata is the second most common pathogen. Despite their high cost, molecular methods have high value in accurate diagnosis of the RVVC.
\end{abstract}

condition, not only affects health of women, but also imposes a considerable financial expenditure and complications for hassle free sexual intercourse, and may even give rise to subfertility (7).

Candida is a genus of yeasts and is the

\footnotetext{
Copyright $(\odot 2021$ The Author(s); Published by Nickan Research Institute. This is an open-access article distributed under the terms of the Creative Commons Attribution License (http://creativecommons.org/licenses/by/4.0), which permits unrestricted use, distribution, and reproduction in any medium, provided the original work is properly cited.
} 
common cause of infections caused by fungi all over the world. It has been reported that this species of fungi are associated with the normal flora of vagina in $20 \%$ to $50 \%$ of women without any clinical sign and symptoms (8). Previous epidemiological studies have shown that Candida albicans is the most common species of fungi that triggered vulva and vagina(9). Candida parapsilosis, Candida krusei, and Candida glabrata are some of the other Candida species that presence of them in the vulvovaginal region has been reported(11). Moreover, Candida albicans plays a crucial role in the pathogenesis of RVVC and its management requires considerable attention to various molecular aspects of it. Genetic factors, sufficient vulvovaginal defense immune mechanism, resistance to antifungal medications, and cultural class are some of the other parameters that must be considered for determining susceptibility to RVVC (12).

\section{Objectives}

The differentiation of varied species of Candida in the samples obtained from vulvovaginal region is imperative. In the present study to gain the up-to-date information on involved species and the prevalence of the RVVC in Isfahan, Iran, we assessed the etiologic agents of aforementioned disease in women referred to the AlZahra hospital (Isfahan, Iran). Furthermore, we surveyed the possible relationship between age, education and marital status with the prevalence of albicans and nonalbicans candidiasis.

\section{Patients and Methods}

\section{Patients and study design}

The present cross-sectional study was performed in AlZahra hospital of Isfahan University of Medical Sciences (MUI), Isfahan, Iran. Our study was conducted on subjects who were admitted to the gynecology and midwifery clinic between September 2017 and August 2018 and had clinical presentations of vulvovaginitis. Demographic information of all patients was documented in special forms. The acceptable criteria for cases to enter the study were patient satisfaction, occurrence of at least four episodes of VVC in previous 12 months, not taking immunosuppressive drugs, and not suffering from diabetes mellitus. Exclusion criteria were occurrence of less than four episodes of VVC in previous year, consumption of immunosuppressive drugs, and suffering from diabetes. Subjects who experienced abnormal vaginal secretions and itch at least four times in the previous 12 months were considered as RVVC and sampling of 100 of them was conducted using sterile swab. Samples were transferred to the hospital lab for further investigation.

\section{Culture media}

We were used Sabouraud dextrose agar and CHROMagar medium (CHROMagar Company, France) as a culture media. Inoculation of the cultures was done at $37^{\circ} \mathrm{C}$ and examined after two days. Based on the instruction of aforementioned culture medium, Candida species were distinguished based on some features such as green color and appearance. In addition to the CHROMagar method, Candida species of fungi were recognized by production of chlamydospore on Corn Meal Agar and formation of germ tube in serum described by Yucesoy et al (10). Absorption of carbohydrate was examined using the API 20C-AUX kit based on the manufacturer's instructions (bioMérieux, Paris, France). As briefly, API 20C stripes were inoculated and incubated at $30^{\circ} \mathrm{C}$ and scored by two blinded observers at 1,2, and 3 days. Reading of reaction was done by referring to the analytical profile index.

\section{Real time-polymerase chain reaction (real time-PCR)}

Deoxyribonucleic acid (DNA) extraction was performed for each sample using the DNA-Free-RY kit (Triplex International Bioscience, China) based on its instructions. Extracted DNA was transferred to the $-20^{\circ} \mathrm{C}$ freezer, with the concentration being measured with NanoDrop (Thermo Scientific) at 280/260 $\mathrm{nm}$. The used primers in the present study were designed according to sequences from the NCBI database and its specificity checked by use of NCBI BLAST tool, primers with no meaningful similarity to other loci were selected. The following primers were used:

- 5'- GGCGCTCAAGCCTGCTACT 3' (Foreword primer for Candida albicans) and 5'-ACGCCTGCCTACTCGTGAA 3' (Reverse primer for Candida albicans),

- 5'-CATTTGCTCAATTGTATGCTGATTT 3' (Foreword primer for Candida parapsilosis) and 5'-CGCAGCATCACGTTTCCA 3' (Reverse primer for Candida parapsilosis),

- 5'-CGAGAACAACTCCGATTCTATAACC 3' (Foreword primer for Candida glabrata) and 5' -TGGACTCAGAATCAGCATTTTCA 3' (Reverse primer for Candida glabrata),

- 5'-CGGCCGGGTCTTTCCTT $\quad 3^{\prime}$ (Foreword primer for Candida krusei) and 5'-CCTGCTTTGAACACTCTAATTTCCT 3' (Reverse primer for Candida krusei).

The molecular assay was done by using Gene Amp 7500 Sequence Detection System (Applied Biosystems, Foster City, CA, USA). To identify each of the four Candida species, $40 \mu \mathrm{L}$ of the PCR reaction mixtures were set up based on the Zhai et al protocol (13). The assessment of sensitivity of method was done by serially diluting template genomic DNA that was prepared from standard Candida cultures. For definitive diagnosis of four Candida species, the extracted DNA from the cultures, reaching 106 colony-forming units $\mathrm{mL}^{-1}$.

\section{Ethical issues}

The present study followed the tenets of the Helsinki Declaration. The ethics committee of Isfahan University 
of Medical Sciences (MUI) accepted this study and the institutional ethical committee at MUI permitted all study protocols (IR.MUI.MED.REC.1398.112). The current study was extracted from the residential thesis of Azadeh Moshtzan at the department of infectious diseases of MUI (Thesis \# 398138).

\section{Statistical analysis}

In the present study, for statistical analyzing chi-square and independent $t$ test was conducted using the SPSS statistical software, (version 16.0, Chicago, IL, USA). A $P$ value $<0.05$ was considered as statistically meaningful.

\section{Results}

Patients $(n=100)$ were evaluated with molecular methods for identification of etiologic agents of RVVC. Based on our statistical analyses, Candida albicans was the main pathogen involved in the pathogenesis of RVVC and Candida glabrata is the second most common pathogen. Moreover, none of the 100 cases that we tested were infected with Candida krusei. Figure 1 shows how the candidiasis species is distributed in patients with RVVC

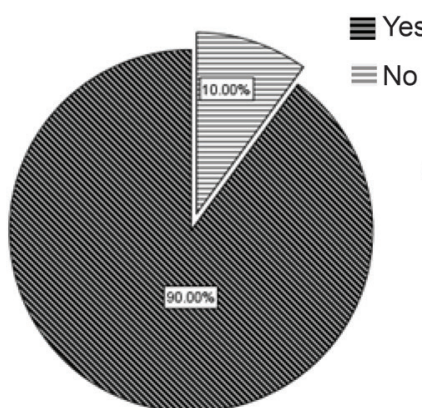

(A) Candida albicans

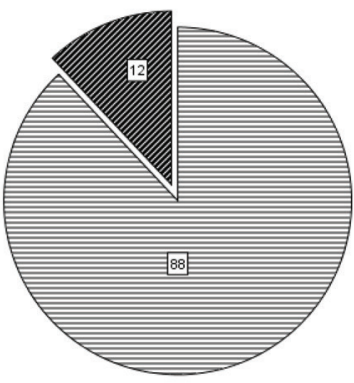

(C) Candida parapsilosis

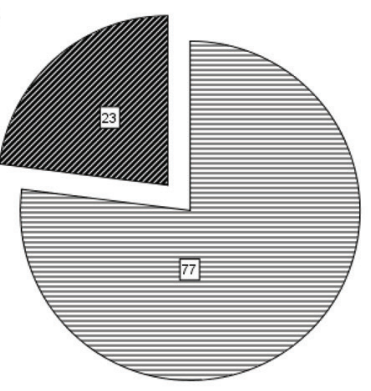

(B) Candida glabrata

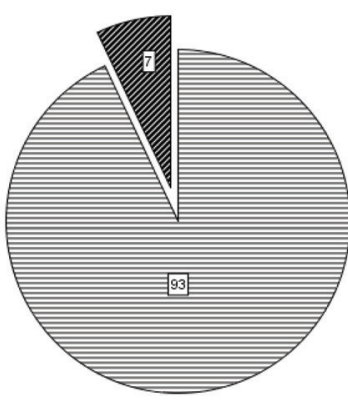

(D) No fungus has grown
Figure 1. Distribution of Candidiasis species in patients with RVVC representations. representations.

In the present study, we found a meaningful relationship between age and RVVC that triggered by Candida albicans $(P<0.05)$; however, there was no significant relationship between age and RVVC that triggered by other fungi such as Candida glabrata, Candida parapsilosis and Candida krusei $(P>0.05$; Table 1$)$.

Table 2 summarizes the results of our study about possible relationship between levels of education and RVVC. There was no meaningful correlation between levels of education and RVVC triggered by various albicans and non-albicans candidiasis $(P>0.05)$.

One of the other parameters evaluated in the present study was the childbirth status. Based on the performed statistical analysis, there was no significant difference between number of children and types of delivery with RVVC triggered by various albicans and non-albicans candidiasis $(P>0.05)$ (Table 3 and Table 4$)$.

\section{Discussion}

As we know, candidiasis is one of the varied infections associated with a range of disease from simple vaginitis to possibly life-threatening conditions such as malignancies $(14,15)$. Involvement of reproductive system, especially external genital organs, is one of the most common presentations due to the above pathogens. VVC results from unusual growth of various types of Candida and affects millions of women in all societies (16). Management of aforementioned condition, especially RVVC, is a puzzling issue for infectious disease specialists because available treatments for aforementioned fungal infections do not work for all women. Among the various sub-types of candidiasis that each year is increasing their variety, Candida albicans was stated as the most common agent of $\operatorname{VVC}(17,18)$; however the issue is open to discussion. In the present study, we evaluated RVVC and the frequency of various species of Candida in subjects from Isfahan, Iran. So far some studies investigated the various aspects and prevalence of candidiasis in Iran. In our study, statistical analyses revealed that Candida albicans was the main pathogen involved in the pathogenesis of RVVC and Candida glabrata is the second most common pathogen. In the present study, we found a significant relationship between age and RVVC triggered by Candida albicans $(P<0.05)$. The mean age of RVVC cases with positive Candida albicans was 32.36 years. This finding is in line with previous reports that considered the crucial role of hormonal alterations in the pathogenesis of VVC. Fidel et

Table 1. Relationship between age and RVVC that triggered by various sub-types of Candida

\begin{tabular}{|c|c|c|c|c|c|c|c|c|}
\hline & \multicolumn{8}{|c|}{ Fungi } \\
\hline & \multicolumn{2}{|c|}{ Candida albicans } & \multicolumn{2}{|c|}{ Candida glabrata } & \multicolumn{2}{|c|}{ Candida parapsilosis } & \multicolumn{2}{|c|}{ Candida krusei } \\
\hline & No & Yes & No & Yes & No & Yes & No & Yes \\
\hline Age $($ mean $\pm \mathrm{SD})$ & $28.3 \pm 4.1$ & $32.36 \pm 6.29$ & $32.04 \pm 6.4$ & $31.6 \pm 5.3$ & $32.07 \pm 6.4$ & $31.08 \pm 4.37$ & $31.9 \pm 6.18$ & - \\
\hline$P$ value & \multicolumn{2}{|c|}{0.04} & \multicolumn{2}{|c|}{0.79} & \multicolumn{2}{|c|}{0.6} & \multicolumn{2}{|c|}{ Not estimate } \\
\hline
\end{tabular}


Table 2. Relationship between levels of education and RVVC

\begin{tabular}{|c|c|c|c|c|c|c|c|c|}
\hline \multirow{3}{*}{ Education } & \multicolumn{8}{|c|}{ Fungi } \\
\hline & \multicolumn{2}{|c|}{ Candida albicans } & \multicolumn{2}{|c|}{ Candida glabrata } & \multicolumn{2}{|c|}{ Candida parapsilosis } & \multicolumn{2}{|c|}{ Candida krusei } \\
\hline & No & Yes & No & Yes & No & Yes & No & Yes \\
\hline Elementary education & 0 & $1(1.1 \%)$ & 0 & $1(4.3 \%)$ & $1(1.1 \%)$ & 0 & $1(1 \%)$ & 0 \\
\hline Middle and high school education & 0 & $10(11.1 \%)$ & $9(11.7 \%)$ & $1(4.3 \%)$ & $9(10.2 \%)$ & $1(8.3 \%)$ & $10(10 \%)$ & 0 \\
\hline Diploma and associate degree & $7(70 \%)$ & $38(42.2 \%)$ & $36(46.8 \%)$ & $9(39.1 \%)$ & $43(48.9 \%)$ & $2(16.7 \%)$ & $45(45 \%)$ & 0 \\
\hline Bachelor's degree & $3(30 \%)$ & $32(35.6 \%)$ & $27(35.1 \%)$ & $8(34.8 \%)$ & $28(31.8 \%)$ & $7(58.3 \%)$ & $35(35 \%)$ & 0 \\
\hline Master's degree & 0 & $9(10 \%)$ & $5(6.5 \%)$ & $4(17.4 \%)$ & $7(8 \%)$ & $2(16.7 \%)$ & $9(9 \%)$ & 0 \\
\hline$P$ value & \multicolumn{2}{|c|}{0.42} & \multicolumn{2}{|c|}{0.14} & \multicolumn{2}{|c|}{0.22} & \multicolumn{2}{|c|}{ Not estimate } \\
\hline
\end{tabular}

Table 3. Relationship between children number and RVVC

\begin{tabular}{|c|c|c|c|c|c|c|c|c|}
\hline \multirow{3}{*}{ Number of children } & \multicolumn{8}{|c|}{ Fungi } \\
\hline & \multicolumn{2}{|c|}{ Candida albicans } & \multicolumn{2}{|c|}{ Candida glabrata } & \multicolumn{2}{|c|}{ Candida parapsilosis } & \multicolumn{2}{|c|}{ Candida krusei } \\
\hline & No & Yes & No & Yes & No & Yes & No & Yes \\
\hline 0 & $2(20 \%)$ & $8(8.9 \%)$ & $9(11.7 \%)$ & 1 & 8 & 2 & 10 & 0 \\
\hline 1 & $7(70 \%)$ & $40(44.4 \%)$ & $36(46.8 \%)$ & 11 & 41 & 6 & 47 & 0 \\
\hline 2 & $1(10 \%)$ & $29(32.2 \%)$ & $22(28.6 \%)$ & 8 & 27 & 3 & 30 & 0 \\
\hline 3 & 0 & $13(14.4 \%)$ & $10(13 \%)$ & 3 & 12 & 1 & 13 & 0 \\
\hline$P$ value & \multicolumn{2}{|c|}{0.15} & \multicolumn{2}{|c|}{0.75} & \multicolumn{2}{|c|}{0.8} & \multicolumn{2}{|c|}{ Not estimate } \\
\hline
\end{tabular}

Table 4. Relationship between types of delivery and RVVC

\begin{tabular}{|c|c|c|c|c|c|c|c|c|}
\hline \multirow{3}{*}{ Types of delivery } & \multicolumn{8}{|c|}{ Fungi } \\
\hline & \multicolumn{2}{|c|}{ Candida albicans } & \multicolumn{2}{|c|}{ Candida glabrata } & \multicolumn{2}{|c|}{ Candida parapsilosis } & \multicolumn{2}{|c|}{ Candida krusei } \\
\hline & No & Yes & No & Yes & No & Yes & No & Yes \\
\hline 0 & 2 & 8 & 9 & 1 & 8 & 2 & 10 & 0 \\
\hline Natural childbirth & 3 & 51 & 44 & 10 & 47 & 7 & 54 & 0 \\
\hline Cesarean & 5 & 31 & 24 & 12 & 33 & 3 & 36 & 0 \\
\hline$P$ value & \multicolumn{2}{|c|}{0.23} & \multicolumn{2}{|c|}{0.15} & \multicolumn{2}{|c|}{0.57} & \multicolumn{2}{|c|}{ Not estimate } \\
\hline
\end{tabular}

al proposed that estrogen, but not progesterone, decreases the ability of epithelium of vulvovaginal tissue to inhibit the growth of Candida albicans (19). In another study, Fischer et al reported that most patients with RVVC were in the perimenopausal age ( 41 to 55 years) and presence of this disease in patients over 50 years of age may be due to exogenous estrogen intake (20). In the current study, there was no significant relationship between age and RVVC that triggered by other fungi such as Candida g glabrata, Candida parapsilosis and Candida krusei $(P>0.05)$. It seems that hormonal alterations performed in childbearing ages were not associated with growth of non-albicans Candida in vulvovaginal tissue; however, for realistic statement, more cellular and molecular studies are necessary.

In the present cross-sectional study, there was no significant difference between number of children and types of delivery with RVVC triggered by various albicans and non-albicans candidiasis. However, the prevalence of Candida albicans was higher in patients who were pregnant (21). Some scientific reports presented that VVC occurrence is frequently more severe during gestation. In the other word; pregnant women are more likely to experience representations of VVC. The main reason for aforementioned event is unclear but it seems that an alteration in mother's immune system is associated with infection susceptibility. Some epidemiological studies have reported a 30\% vaginal candidosis outbreak among pregnant women, especially during the 2 nd and 3 rd trimester of pregnancy (22). In our study, number of pregnancy in RVVC patients infected with Candida albicans was higher than those infected with non-albicans Candida. The main reason for this result is not clear and more molecular investigations are required.

\section{Conclusion}

Candida albicans was the main pathogen involved in the pathogenesis of RVVC in Isfahan while Candida glabrata was the second most common pathogen. Despite their high cost, molecular methods have high value in accurate diagnosis of the RVVC.

Limitations of the study

The small sample of the subjects and also single-center study were the considerable limitations of the present investigation. 


\section{Authors' contribution}

In the present cross-sectional study, KS and ZA were the principal investigators. AM, KS, and ZA contributed to designing the study. AM participated in writing the initial format of the manuscript. AM, KS, and ZA confirmed the accuracy of any part of the present manuscript.

\section{Conflicts of interest}

The authors of present manuscript declare that there are no conflicts of interest.

Ethical considerations

Ethical issues (including plagiarism, data fabrication, double publication) have been completely observed by the authors.

\section{Funding/Support}

The present study was supported financially by MUI Deputy Research, Isfahan, Iran (Grant \# 398138).

\section{References}

1. Willems HM, Ahmed SS, Liu J, Xu Z, Peters BM. Vulvovaginal candidiasis: a current understanding and burning questions. J. Fungus. 2020;6:27. doi: 10.3390/jof6010027.

2. Gonçalves B, Ferreira C, Alves CT, Henriques M, Azeredo J, Silva S. Vulvovaginal candidiasis: Epidemiology, microbiology and risk factors. Crit Rev Microbiol. 2016;42:905-27. doi: 10.3109/1040841X.2015.1091805

3. Yano J, Sobel JD, Nyirjesy P, Sobel R, Williams VL, Yu Q, et al. Current patient perspectives of vulvovaginal candidiasis: incidence, symptoms, management and post-treatment outcomes. BMC Womens Health. 2019;19:48. doi: 10.1186/ s12905-019-0748-8.

4. Sobel JD. Recurrent vulvovaginal candidiasis. Am J Obstet Gynecol. 2016;214:15-21. doi: 10.1016/j.ajog.2015.06.067.

5. Blostein F, Levin-Sparenberg E, Wagner J, Foxman B. Recurrent vulvovaginal candidiasis. Ann Epidemiol. 2017;27:575-82. e3. doi: 10.1016/j.annepidem.2017.08.010.

6. Denning DW, Kneale M, Sobel JD, Rautemaa-Richardson R. Global burden of recurrent vulvovaginal candidiasis: a systematic review. Lancet Infect Dis. 2018;18:e339-47. doi: 10.1016/S1473-3099(18)30103-8.

7. Pellati D, Mylonakis I, Bertoloni G, Fiore C, Andrisani A, Ambrosini G, et al. Genital tract infections and infertility. Eur J Obstet Gynecol Reprod Biol. 2008;140:3-11. doi: 10.1016/j. ejogrb.2008.03.009.

8. Vermitsky J-P, Self MJ, Chadwick SG, Trama JP, Adelson ME, Mordechai E, et al. Survey of vaginal-flora Candida species isolates from women of different age groups by use of speciesspecific PCR detection. J Clin Microbiol. 2008;46:1501-3. doi: 10.1128/JCM.02485-07

9. Ghaddar N, Anastasiadis E, Halimeh R, Ghaddar A, Dhar R, AlFouzan $W$, et al. Prevalence and antifungal susceptibility of Candida albicans causing vaginal discharge among pregnant women in Lebanon. BMC Infectious Diseases. 2020 Dec;20(1):1-9. doi: 10.1186/s12879-019-4736-2.

10. Yucesoy M, Marol S. Performance of CHROMAGAR Candida and BIGGY agar for identification of yeast species. Annal Clin Microbiol Antimicrob 2003;2:1-7. doi: 10.1186/1476-07112-8.

11. Cheng M-F, Yang Y-L, Yao T-J, Lin C-Y, Liu J-S, Tang R-B, et al. Risk factors for fatal candidemia caused by Candida albicans and non-albicans Candida species. BMC Infect Dis. 2005;5:22. doi: 10.1186/1471-2334-5-22.

12. Davidson L, Netea M, Kullberg B. Patient susceptibility to candidiasis-a potential for adjunctive immunotherapy. Fungus. 2018;4:9. doi: 10.3390/jof4010009.

13. Zhai Y, Liu J, Zhou L, Ji T, Meng L, Gao Y, et al. Detection of Candida species in pregnant Chinese women with a molecular beacon method. J Med Microbiol. 2018;67: 783.doi: 10.1099/ jmm.0.000740.

14. Hachem R, Hanna H, Kontoyiannis D, Jiang $Y$, Raad I. The changing epidemiology of invasive candidiasis: Candida glabrata and Candida krusei as the leading causes of candidemia in hematologic malignancy. Cancer. 2008;112:2493-9. doi: 10.1002/cncr.23466.

15. Bansal R, Pallagatti S, Sheikh S, Aggarwal A, Gupta D, Singh R. Candidal species identification in malignant and potentially malignant oral lesions with antifungal resistance patterns. Contemp Clin Dent. 2018;9:S309. doi: 10.4103/ccd. ccd_296_18.

16. Rosati D, Bruno M, Jaeger M, ten Oever J, Netea MG. Recurrent vulvovaginal candidiasis: an immunological perspective. Microorganisms. 2020;8:144. doi: 10.3390/ microorganisms8020144.

17. Mayer FL, Wilson D, Hube B. Candida albicans pathogenicity mechanisms. Virulence. 2013;4:119-28. doi: 10.4116/ viru. 22913

18. Donders GG, Grinceviciene S, Ruban K, Bellen G. Vaginal $\mathrm{pH}$ and microbiota during fluconazole maintenance treatment for recurrent vulvovaginal candidosis (RVVC). Diagn Microbiol Infect Dis . 2020;14:115024. doi: 10.1016/j. diagmicrobio.2020.115024.

19. Fidel PL, Cutright J, Steele C. Effects of reproductive hormones on experimental vaginal candidiasis. Infect Immun. 2000;68:651-7. doi: 10.1128/IAI.68.2.651-657.2000

20. Fischer G, Bradford J. Vulvovaginal candidiasis in postmenopausal women: the role of hormone replacement therapy. J Low Genit Tract Dis. 2011;15:263-7. doi: 10.1097/ LGT.0b013e3182241f1a.

21. Aguin T, Sobel J. Vulvovaginal candidiasis in pregnancy. Curr Infect Dis Rep. 2015;17:30. doi: 10.1007/s11908-015-0462-0.

22. RamoseSilva M, Martins NR, Kroumpouzos G. Oral and vulvovaginal changes in pregnancy. Clin Dermatol. 2016;34(3):353-8. doi: 10.1016/j.clindermatol.2016.02.007. 